\title{
Parásitos en Arapaima gigas de la Amazonía Peruana según Grupo Etario
}

\author{
Parasites in Arapaima gigas from the Peruvian Amazon by Age Group \\ Enrique Serrano-Martínez ${ }^{1,2}$, Manuel Tantaleán V. ${ }^{1}$, Guillermo Leguía P. ${ }^{1}$, \\ Marco Quispe H. ${ }^{1}$, Gina C. Casas V. ${ }^{1}$
}

\section{Resumen}

El presente estudio se realizó en 120 paiches (Arapaima gigas) distribuidos en cuatro grupos etarios ( 10 a 30, 31 a 180, 181 a 365 días y $>1$ año de edad), procedentes de centros de cultivo de la Amazonía peruana para determinar la prevalencia de parasitismo así como los órganos afectados. Se reporta la presencia de ocho especies de parásitos, siendo monogeneos (Dawestrema cycloancistrium y D. cycloancistrioides) en branquias; protozoos (Piscinodinium pillulare, Ichthyophthirius multifiliis y Trichodina sp) en piel, aletas y branquias; digeneos (Caballerotrema brasiliense) en intestino; cestodos (Schizochoerus liguloides) en cavidad abdominal; y nematodos (Nilonema senticosum) en vejiga natatoria. La prevalencia de parasitismo global fue de $0,66.7,90.0$ y $66.7 \%$ para los grupos etarios de 10-30, 31-180, 181-365 días y >1 año de edad, respectivamente.

Palabras clave: Arapaima, cultivo, parásitos, Amazonía peruana

\section{Abstract}

This study was carried out in 120 paiches (Arapaima gigas) distributed in four age groups (10 to 30,31 to 180,181 to 365 days and $>1$ year of age) from fish farms in the Peruvian Amazon to determine the prevalence and the affected organs. Eight parasite species are reported: monogeneans (Dawestrema cycloancistrium and $D$. cycloancistrioides), digeneans (Caballerotrema brasiliense), protozoa (Piscinodinium pillulare, Ichthyophthirius multifiliis and Trichodina sp), cestodes (Schizochoerus liguloides), and nematodes (Nilonema senticosum). The prevalence of parasitism was 0 , $66.7,90.0$ and $66.7 \%$ for the age groups $10-30,31-180,181-365$ days and $>1$ year of age, respectively.

Key words: Arapaima gigas, aquaculture, parasites, Peruvian Amazon

\footnotetext{
${ }^{1}$ Grupo SALUVET-UPCH, Facultad de Medicina Veterinaria y Zootecnia, Universidad Peruana Cayetano Heredia, Lima, Perú

${ }^{2}$ E-mail: enrique.serrano@upch.pe
}

Recibido: 19 de agosto de 2014

Aceptado para publicación: 11 de diciembre de 2014 


\section{INTRODUCCIÓN}

El paiche Arapaima gigas es uno de los dos peces más grandes de América del Sur, pudiendo llegar a medir más de $2 \mathrm{~m}$ de longitud y pesar más de $100 \mathrm{~kg}$. Es una especie de importancia en la economía pesquera del Amazonas. Su rápido crecimiento en el primer año de cultivo y su gran aceptación en el mercado ha favorecido su crianza comercial (Salvo-Souza y Val, 1990).

En el Perú, la pesca del paiche por parte del poblador amazónico, como especie de consumo y generador de ingresos a través de la comercialización de sus productos, propició una intensa captura en su medio natural, causando una severa disminución del desembarque (de 700 a $144 \mathrm{TM}$ ) en las últimas dos décadas en la región de Loreto, de allí que en la actualidad se le considera especie en peligro de extinción (Apéndice II, de la Convención Internacional de Especies Amenazadas de Fauna y Flora-CITES-). Debido a ello, las autoridades locales y regionales han iniciado una exhaustiva promoción del cultivo de esta especie en ambientes controlados.

El paiche es una especie de hábitos alimenticios piscívoros. Inicia su desarrollo con la ingestión de crustáceos y moluscos y a partir del año de edad consume peces (Queiroz y Sardinha, 1999; Imbiriba, 2001). El alimento que ingiere puede actuar como hospedero intermediario de varias especies parasitarias, afectado su estado sanitario. En el país, Mathews et al. (2007) reportaron la presencia de parásitos externos como Dawestrema cycloancistrium, Trichodina fariai e Ichthyophthirius multifilis en alevinos de paiche, en tanto que Cotrina y Rebaza (2008) reportaron Trichodina sp, D. cycloancistrioides, D. cycloancistrium, Dolops sp, Nilonema senticosum, Caballerotrema sp y Gymnodinium sp en juveniles de paiche criados en cautiverio. Sin embargo, no existen mayores reportes que permitan elaborar programas de manejo sanitario para prevenir la presentación de parásitos y con ello mejorar la producción de esta especie. Por ello, el presente estudio se abocó a determinar la presencia de parásitos en paiches procedentes de la acuicultura, evaluando las etapas de desarrollo con mayor prevalencia de parásitos.

\section{Materiales y Métodos}

\section{Lugar de Estudio y Especímenes}

El estudio se llevó a cabo con paiches de dos centros de cultivo localizados en la Carretera Iquitos-Nauta, región Loreto, Perú. Las muestras fueron trabajadas en el Laboratorio de Parasitología de la Facultad de Medicina Veterinaria y Zootecnia (FAVEZ), Universidad Peruana Cayetano Heredia (UPCH), Lima.

Entre mayo de 2009 y enero de 2011 se muestrearon 120 paiches de diversos estadios de desarrollo. Para efecto del estudio, los peces se clasificaron en cuatro grupos etarios ( 10 a 30,31 a 180, 181 a 365 días y más de 1 año de edad), con tallas promedio de $7,16,40$ y $104 \mathrm{~cm}$ y pesos promedio de $7.7,38.7,635$ y $2246 \mathrm{~g}$, respectivamente. El número de paiches trabajado por centro de cultivo fue similar.

\section{Colección y Procesamiento de Muestras}

Mediante microscopía óptica se observaron raspados de piel y aletas, que fueron colocados en láminas portaobjetos y cubiertos con laminillas cubreobjetos para la identificación de protozoarios ectoparásitos.

Las branquias, seccionadas para separar los arcos, se colocaron en una placa de Petri con agua y se observaron en un microscopio estereoscópico para la búsqueda de monogeneos. Una vez detectados, se colocaron en una lámina portaobjetos con una gota de agua y se cubrió con una laminilla; 
donde se agregó picrato de amonio y glicerina para estudiar las estructuras quitinosas del opistohaptor y del complejo genital masculino (Malmberg, 1957).

En la cavidad abdominal, se observó la superficie de las vísceras y la vejiga natatoria en busca de helmintos. El estómago e intestino fueron colocados en placas de Petri conteniendo agua corriente para el examen del contenido previa disección. Una vez obtenidos los parásitos, se lavaron y se fijaron en formol (cestodos) o alcohol de $70 \%$ caliente (nematodos). Para la identificación, los céstodos fueron coloreados con carmín y los nematodos fueron clarificados en una solución de alcohol-fenol.

En el caso de trematodos, se lavaron y fijaron en formol al 10\%, previo aplanamiento entre lámina y laminilla por 24 horas, y se colorearon con carmín acético de Semichon. Luego fueron deshidratados, clarificados y montados en preparaciones permanentes con bálsamo de Canadá (Eiras et al., 2002).

La identificación de las formas parasitarias fue realizada con el apoyo de los trabajos realizados por Thatcher (1980), Kristsky et al. (1985), Gibson (1994), Costadinova y Gibson (2001), Moravec et al. (2006) y Miranda et al. (2012).

Las muestras de heces fueron sometidas a técnicas coproparasitológicas de rutina, incluyendo frotis y coloración con tricrómica de Gomori. Los análisis hemoparasitológicos se realizaron mediante frotis sanguíneo y coloración con Giemsa y microcapilar. Los hallazgos se documentaron fotográficamente y el material se encuentra archivado y codificado en el Laboratorio de Parasitología de la FAVEZ-UPCH.

\section{Análisis de Datos}

Los valores de prevalencia, intensidad media de infección y abundancia media se calcularon según Bush et al. (1997).
Resultados

En los 120 peces muestreados se identificaron parásitos monogeneos (Dawestrema cycloancistrium y D. cycloancistrioides) y protozoos (Piscinodinium pillulare, Ichthyophthirius multifiliis y Trichodina sp) en piel, aletas y branquias; así como digeneos (Caballerotrema brasiliense) en intestino, cestodos (Schizochoerus liguloides) en cavidad abdominal y nematodos (Nilonema senticosum) en vejiga natatoria (Cuadro 1).

El grupo etario con mayor prevalencia de parásitos fue aquel entre 181 y 365 días de edad (90\%), siendo nula la presencia de parásitos en la etapa de 10 a 30 días de edad (Cuadro 2). De los parásitos hallados, se resalta la presencia del monogeneo Dawestrema sp en peces de 31 a 180 días de edad, de protozoos $P$. pillulare, I. multifiliis y Trichodina sp en peces mayores de 30 días de edad, y de digeneos ( $C$. brasiliense), cestodos ( $S$. liguloides) y nematodos $(N$. senticosum) en paiches de más de un año de edad (Cuadro 3).

\section{Discusión}

El estudio demuestra la presencia de ocho especies de parásitos, siendo cuatro en piel, aleta y branquias, una en branquias y tres en intestino, vejiga natatoria y cavidad abdominal; donde su presentación estuvo asociada a la edad o estadio de desarrollo de los peces. Las especies identificadas fueron también reportadas por Thatcher (2006) y Dos Santos et al. (2008) en paiches provenientes del río Amazonas en Brasil.

En relación a parásitos protozoarios apicomplexos, Bonar et al. (2006) reportan la presencia de Calyptospora sp en paiches juveniles exportados desde Brasil hacia los Estados Unidos de Norteamérica. Asimismo, se han identificado otras especies de Calyptospora como C. serrasalmi en hígado y célula pancreática acinar intrahepática 
Cuadro 1. Prevalencia y localización de parásitos en paiche Arapaima gigas $(\mathrm{n}=120)$ procedentes de centros de cultivo de la provincia de Maynas, región Loreto, Perú

\begin{tabular}{|c|c|c|c|}
\hline \multirow{2}{*}{ Parásito } & \multicolumn{2}{|c|}{ Positivos } & \multirow{2}{*}{ Ubicación } \\
\hline & (n) & $(\%)$ & \\
\hline \multicolumn{4}{|l|}{ Monogenea } \\
\hline $\begin{array}{l}\text { Dawestrema } \\
\text { cycloancistrium }\end{array}$ & 46 & 38.3 & Piel, aletas y branquias \\
\hline $\begin{array}{l}\text { Dawestrema } \\
\text { cycloancistrioides }\end{array}$ & 1 & 0.8 & Branquias \\
\hline \multicolumn{4}{|l|}{ Digenea } \\
\hline $\begin{array}{l}\text { Caballerotrema } \\
\text { brasiliense }\end{array}$ & 2 & 1.7 & Intestino \\
\hline \multicolumn{4}{|l|}{ Protozoo } \\
\hline $\begin{array}{l}\text { Piscinodinium } \\
\text { pillulare }\end{array}$ & 9 & 7.5 & Piel, aletas y branquias \\
\hline $\begin{array}{l}\text { Ichthyophthirius } \\
\text { multifiliis }\end{array}$ & 5 & 4.2 & Piel, aletas y branquias \\
\hline Trichodina $\mathrm{sp}$ & 13 & 10.8 & Piel, aletas y branquias \\
\hline \multicolumn{4}{|l|}{ Cestodo } \\
\hline $\begin{array}{l}\text { Schizochoerus } \\
\text { liguloides }\end{array}$ & 2 & 1.7 & Cavidad abdominal \\
\hline \multicolumn{4}{|l|}{ Nematodo } \\
\hline Nilonema senticosum & 18 & 15.0 & Vejiga natatoria \\
\hline
\end{tabular}

Cuadro 2. Prevalencia de parásitos en paiche Arapaima gigas, según grupo etario (30 peces por grupo)

\begin{tabular}{cc}
\hline $\begin{array}{l}\text { Estadio de } \\
\text { desarrollo }\end{array}$ & $\begin{array}{c}\text { Prevalencia } \pm \\
\text { I.C. }\end{array}$ \\
\hline 10-30 días & 0 \\
31-180 días & $66.7 \pm 16.9$ \\
181-365 días & $90.0 \pm 10.7$ \\
$>1$ año & $66.7 \pm 16.9$ \\
\hline Total & $55.8 \pm 17.8$ \\
\hline
\end{tabular}

${ }^{1}$ Intervalo de confianza del $95 \%$ de la piraña negra (Serrasalmus niger) (Cheung et al., 1986) y C. tucunarensis en hígado de tucunaré (Cichla ocellaris), provenientes del medio natural y de cultivo (Békési y Molnár, 1991). Araújo et al. (2009), reportaron la presencia de Trichodina sp e Ichthyobodo sp en Brasil. En el presente estudio se hallaron tres especies de protozoos (Piscinodinium pillulare, Ichthyophthirius multifiliis y Trichodina sp).

Estudios realizados en Brasil reportan la presencia de nematodos en paiches, como Capillostrongyloides arapaimae en intestino y ciego pilórico (Santos et al., 2008), 
Cuadro 3. Prevalencia (P), intensidad media (IM) y abundancia media (AM) de parásitos de paiche Arapaima gigas, procedentes de centros de cultivo de la provincia de Maynas, Región Loreto, según grupo etario ${ }^{1}$

\begin{tabular}{lcccccccccc}
\hline \multirow{2}{*}{ Parásito } & \multicolumn{3}{c}{$31-180$ días } & \multicolumn{3}{c}{$181-365$ días } & \multicolumn{3}{c}{$>1$ año } \\
\cline { 2 - 10 } & $\mathrm{P}$ & $\mathrm{IM}$ & $\mathrm{AM}$ & $\mathrm{P}$ & $\mathrm{IM}$ & $\mathrm{AM}$ & $\mathrm{P}$ & $\mathrm{IM}$ & AM \\
\hline D. cycloancistrium & 63.3 & 3.2 & 2.1 & 90.0 & 2.2 & 2 & 0 & 0 & 0 \\
D. cycloancistrioides & 3.3 & 63 & 2.1 & 0 & 0 & 0 & 0 & 0 & 0 \\
C. brasiliense & 0 & 0 & 0 & 0 & 0 & 0 & 6.7 & 15 & 1 \\
P. pillulare & 0 & 0 & 0 & 26.7 & 12.8 & 3.4 & 3.3 & 60 & 2 \\
I. multifiliis & 13.3 & 2 & 0.27 & 3.3 & 5 & 0.2 & 0 & 0 & 0 \\
Trichodina sp. & 43.3 & 2 & 0.87 & 0 & 0 & 0 & 0 & 0 & 0 \\
S. liguloides & 0 & 0 & 0 & 0 & 0 & 0 & 6.7 & 2 & 0.1 \\
N. senticosum & 0 & 0 & 0 & 0 & 0 & 0 & 60 & 1.7 & 1 \\
\hline
\end{tabular}

${ }^{1}$ El grupo etario de 10 a 30 días de edad no presentó parásitos

Camallanus tridentatus en estómago, ciego e intestino (Araújo et al., 2009; Santos y Moravec, 2009a), Terranova serrata en estómago e intestino (Araújo et al., 2009), Goezia spinulosa en estómago (Dos Santos et al., 2008; Santos y Moravec, 2009b), Nilonema senticosum en vejiga natatoria (Moravec et al., 2006; Santos y Gibson, 2007; Dos Santos et al., 2008; Santos y Moravec, 2009c) y Rumai rumai en boca, lengua, opérculo y cabeza (Santos y Moravec, 2009c). En el presente estudio se encontró únicamente Nilonema senticosum.

Los monogeneos (Dawestrema cycloancistrium y D. cycloancistrioides) y digeneos (Caballerotrema brasiliense) hallados en el presente estudio concuerdan con hallazgos de Araújo et al. (2009) y Dos Santos et al. (2008). También se ha reportado la presencia de acantocéfalos como el Polyacanthorhynchus rhopalorhynchus en paiches (Dos Santos et al., 2008).
La nula presencia de parásitos en alevinos de 10 a 30 días contradice lo hallado por Mathews et al. (2007), quienes reportaron la presencia de Dawestrema cycloancistrium, Trichodina fariai e Ichthyophthirius multifiliis en alevinos. En el presente estudio, el parasitismo se observó a partir de los 32 días de edad, destacando Dawestrema, Nilonema y Trichodina, lo cual es corroborado por Cotrina y Rebaza (2008) en paiches juveniles donde, además de estos tres parásitos, reportaron Caballerotrema $\mathrm{sp}$ y Gymnodinium sp.

En este sentido, el presente estudio determina la presentación de parásitos dependientes de la etapa de desarrollo del paiche, posiblemente influenciadas por factores estresantes como los cambios de estanques, cambio de alimentación, reproducción, etc., los cuales son más notorios en centros de cultivo que en el medio natural. 
Conclusiones

- Se reporta la presencia de ocho especies de parásitos en la especie Arapaima gigas: Dawestrema cycloancistrium, D. cycloancistrioides (monogeneos), Piscinodinium sp, Ichthyophthirius $\mathrm{sp}$ y Trichodina sp (protozoos) en piel y branquias, Caballerotrema brasiliense (digeneo) en intestino, Nilonema senticosum (nematodo) en vejiga natatoria, y Schizochoerus liguloides (cestodo) en cavidad abdominal.

- La presencia de parásitos varió de acuerdo a la edad de los peces, siendo nula en el grupo de alevinos (menores de 30 días).

\section{Agradecimientos}

Los autores agradecen a los acuicultores Sres. Wenseslao Solsol y Manuel García por el apoyo brindado en campo durante la ejecución del estudio. Del mismo modo, a los profesionales de la Dirección Regional de la Producción (DIREPRO) Loreto, Blgos. César Correa y Debbie Reátegui, por el apoyo durante las coordinaciones en campo y prestación de instalaciones durante el desarrollo del estudio. A la Universidad Nacional de la Amazonía Peruana (UNAP) por la prestación de sus instalaciones de laboratorio. Un especial agradecimiento al Blgo. Carlos Cabrera por su incondicional apoyo como profesional y que Dios lo guarde en su gloria.

El presente trabajo fue desarrollado mediante el apoyo financiero de la Presidencia del Consejo de Ministros a través del FINCyT (Contrato N| 017-FINCyT-PIBAP2009).

\section{Literatura Citada}

1. Araújo CSO, Gomes AL, TavaresDías M, Andrade SMS, Belem-Costa A, Borges JT, et al. 2009. Parasitic infections in pirarucu fry, Arapaima gigas Schinz, 1822 (Arapaimatidae) kept in a semi-intensive fish farm in Central Amazon, Brazil. Veterinarski Archiv 79: 499-507.

2. Békesi L, Molnar K. 1991. Calyptospora tucunarensis n.s.p. (Apicomplexa: Sporozoa) from the liver of tucunare Cichla ocellaris in Brazil. Syst Parasitol 18: 127-132. doi: 10.1007/ BF00017665

3. Bonar C, Poynton S, Schulman Y, Rietcheck R, Garner M. 2006. Hepatic Calyptospora sp. (Apicomplexa) infection in a wild-born, aquarium-held clutch of juvenile arapaima Arapaima gigas (Osteoglossidae). Dis Aquat Org 70: 81-92. doi: 10.3354/dao070081

4. Bush AO, Lafferty KD, Lotz JM, Shostak AW. 1997. Parasitology meets ecology on its own terms: Margolis et al. Revisited. J Parasitol 83: 575-583.

5. Cheung PJ, Nigrelli RF, Ruggieri GD. 1986. Calyptospora serrasalmi $\mathrm{sp} . \mathrm{n}$. (Coccidia: Calyptosporidae) from liver of the black piranha, Serrasalmus niger Schomburgk. J Aquaricult Aquat Sci 4: 54-57.

6. Costadinova A, Gibson D. 2001. Redescriptions of two echinostomes from freshwater fishes, with comments on Singhia Yamaguti, 1958 and Caballerotrema Prudhoe, 1960 (Digenea: Echinostomatidae). Syst Parasitol 49: 195-204.

7. Cotrina M, Rebaza C. 2008. Caracterización de la fauna parasitaria en juveniles de paiche «Arapaima gigas» criados en cautiverio. En: Memoria Institucional 2008. Programa de Investigación en Ecosistemas Acuáticos del IIAP. p 35-36.

8. Dos Santos S, Ceccarelli P, Luque J. 2008. Helmintos parasitos do pirarucu, Arapaima gigas (Schinz, 1822) (Osteoglossiformes: Arapaimidae), no Rio Araguaia, Estado de Mato Grosso, Brasil. Rev Bras Parasitol Vet 17: 171173. doi: 10.1590/S1984-29612008000300012 
9. Eiras JC, Takemoto RM, Pavanelli GC. 2002. Métodos de estúdio y técnicas laboratoriales en parasitologia de peces. Ed. Acribia. España. 142 p.

10. Gibson DI. 1994. Order Amphilinidea Poche 1922. In: Khalil LF, Jones A, Bray RA (eds). Key to the cestode parasites of vertebrates. Wallingford, USA: CAB International. p 3-10.

11. Imbiriba EP. 2001. Potencial de criação de pirarucu, Arapaima gigas, em cativeiro. Acta Amazônica 31: 299-316.

12. Kritsky DC, Boeger A, Thatcher VE. 1985. Neotropical Monogenea. 7. Parasites of the pirarucu, Arapaima gigas (Cuvier, 1829) with descriptions of two news species and redescription of Dawestrema cycloancistrium Price and Nowlin, 1967 (Dactylogyridae: Ancyrocephalinae). P Biol Soc Wash 98: 321-331.

13. Malmberg G. 1957. Om förekomsten av Gyrodactylus pa svenska fisker. Skr Söd Sver Fisk For Arsskr 1956: 19-76.

14. Mathews P, Chu-Koo FW, Oliveira JC, Ismiño R, Gomes AL, Varella AMB, Tello $S$. 2007. Fauna ectoparasitaria en alevinos de paiche Arapaima gigas (Shinz 1822) cultivados en el Centro de Investigaciones de Quistococha. Folia Amazónica 16: 23-27.

15. Miranda LH, Marchiori N, Alfaro CR, Martins ML. 2012. First record of Trichodina heterodentata (Ciliophora: Trichodinidae) from Arapaima gigas cultived in Peru. Acta Amaz 42: 433-438. doi: 10.1590/S0044-59672012000300016

16. Moravec F, Scholz T, Kuchta R, Dykova I, Posel P. 2006. New data on the morphology of Nilonema senticosum (Nematoda, Philometridae), a parasite of Arapaima gigas (Pisces), with notes on another philometrid, Alinema amazonicum, in Peru. Acta Parasitologica 51:279-285.
17. Queiroz HL, Sardinha AD. 1999. Apreservação e o uso sustentado dos pirarucus (Arapaima gigas, Osteoglossidae) em Mamirauá. En: Estratégias para manejo de recursos pesqueiros em Mamirauá. Manaus: Sociedade Civil Mamirauá/MCT/CNPq. p 108-141.

18. Salvo-Souza RH, Val AL. 1990. O gigante das águas doces. Ciên Hoje 11: 9-12.

19. Santos CP, Gibson DI. 2007. Nilonema gymnarchi Khalil, 1960 and $N$. senticosum (Baylis, 1922) (Nematoda: Dracunculoidea): Gondwana relicts. Syst Parasitol 67: 225-234.

20. Santos CP, Moravec F, Venturieri R. 2008. Capillostrongyloides arapaimae sp. n. (Nematoda: Capillariidae), a new intestinal parasite of the arapaima Arapaima gigas from the Brazilian Amazon. Mem Inst Oswaldo Cruz 103: 392-395.

21. Santos CP, Moravec F. $2009 a$. Camallanus tridentatus (Drasche) (Nematoda: Camallanidae): new taxonomically important morphological data. Mem Inst Oswaldo Cruz 104: 93-99.

22. Santos CP, Moravec F. 2009b. Goezia spinulosa (Nematoda: Raphidascarididae), a pathogenic parasite of the arapaima Arapaima gigas (Osteichthyes). Folia Parasitol 56: 55-63.

23. Santos CP, Moravec F. 2009c. Tissuedwelling philometrid nematodes of the fish Arapaima gigas in Brazil. J Helminthol 83: 295-301. doi: 10.1017/ S0022149X09224163

24. Thatcher V. 1980. Duas novas espécies de Caballerotrema (Trematoda: Echinostomatidae) do pirarucu e do aruana (Osteoglossidae), con uma redefinicao do genero e uma redescricao de C. brasiliense Prudhoe, 1960. Acta Amaz 10: 419- 423.

25. Thatcher VE. 2006. Amazon fish parasites. Sofia: Pensoft. 508 p. 